\title{
A SURVEY OF Ha IN THE BRIGHTER NORTHERN BE STARS
}

\author{
WILLIAM P. BIDELMAN and ANTHONY J. WEITENBECK \\ Warner and Swasey Observatory, Case Western Reserve University, Cleveland, Ohio, U.S.A.
}

\begin{abstract}
H} \alpha$ observations have been made of the stars brighter than $m_{v}=7.5$ and north of $\delta=-30^{\circ}$ contained in the three classical Be-star catalogues of Merrill and Burwell. At least one observation of each star was made at each of the two epochs 1958/9 and 1970/2 at the Lick Observatory and at the Warner and Swasey Observatory respectively. The dispersions used were $88 \AA \mathrm{mm}^{-1}$ at Lick and $66 \AA_{\mathrm{mm}}^{-1}$ and $18 \AA \mathrm{mm}^{-1}$ (with image tube) at Warner and Swasey.

Results were as follows: The total number of objects observed was 215 . Of these, 32 were supergiants and will be discussed later. Another 16 stars were deemed abnormal in some manner (helium and/or forbidden emission, composite spectrum, markedly violet-displaced $\mathrm{H} \alpha$ emission component: an interesting group of five stars including the nebular variable AB Aurigae). Thirteen of the stars showed no emission at either epoch. Of the 154 presumably normal low-luminosity stars showing emission, 70 displayed shell structure in $\mathrm{H} \alpha$ at one or both epochs, though in over half of these cases the shell absorption was considered 'weak'. Thirty of the 70 stars varied in their shell characteristics between the two epochs. The total number of stars whose $\mathrm{H} \alpha$ line varied in some respect was 43 , or 26 percent of the 167 normal low-luminosity stars observed. Quite a number of the stars observed have been reported to show shell characteristics by others but were not so noted by us.

Eight additional Be stars not contained in the Merrill-Burwell catalogues have also been observed. Seven of these are shell stars; the other is the unusually high latitude object HD 127617.

It is a pleasure to acknowledge that many of the Lick observations were made by Dr Jack E. Forbes, a graduate student at the time in the Department of Astronomy at the University of California, Berkeley.
\end{abstract}

\section{DISCUSSION}

Conti: I want to be clear as to your definition of a shell. Do you call a shell star one with an $\mathrm{H} \alpha$ line showing double emission and central absorption?

Bidelman: Yes. But you must remember that my dispersion is not particularly high, so any such structure that I see. would no doubt be very conspicuous. at coudé dispersion. Since I have not done spectrophotometry, however, I cannot tell you the depths of the $\mathrm{H} \alpha$ reversals observed, though in most cases they must be as low as the continuum and in some well below it.

Peters: We have been surveying Be stars at $\mathrm{H} \alpha$ using the Lick Observatory cooled $40 \mathrm{~mm}$ Varo image tube. Our dispersions range from $11 \AA \mathrm{mm}^{-1}$ to $33 \AA \mathrm{mm}^{-1}$. One aspect of our project has been to obtain light exposures on baked IIIa-J plates to look for structure in $\mathrm{H} \alpha$. We have found that the profiles of $\mathrm{H} \alpha$ in Be stars are far more complicated than was previously believed to be the case. Often times we observe fine structure which does not persist from one observation to the next.

Bidelman: Your point about making the exposures light is certainly a good one. In many cases, we had to reobserve the star to get an exposure light enough to see structure at $\mathbf{H} \alpha$. It is also true that if the emission is narrow the chance of seeing structure in it is less. I might add that practically every shell star I had has a rotational velocity of at least $300 \mathrm{~km} \mathrm{~s}^{-1}$.

Doazan: Are the variations which you observed based on eye estimates?

Bidelman: Yes. 